concentration above the in vitro sensitivity level for at least three to four days.

Although $\mathrm{Cl}$. tetani infection in mice could effectively be controlled with penicillin, spores could nevertheless survive in the tissues and be reactivated to cause fatal tetanus.

Penicillin was unable to prevent tetanus in mice when given late after infection, at a time when antitoxin was effective.

Experiments in mice showed that penicillin failed to prevent tetanus when the spores were injected together with penicillinaseproducing staphylococci.

It is suggested that non-immune wounded persons who receive penicillin prophylaxis should also be given at the same time an injection of adsorbed tetanus toxoid which would serve as the first dose of a full course of active immunization.

Thanks are due to Dr. H. E. L'Etang, of Wyeth Laboratories, for the gift of both long-acting penicillin preparations.

\section{REFERENCES}

Anwar, A. A., and Turner, T. B. (1956). Bull. fohns Hopk. Hosp., 98, 85 .
Bigger, J. W. (1944). Lancet, 2, 497.

Bliss, E. A., Warth, P. T., and Chandler, C. A. (1950). Ann. N.Y. Acad. Sci., 53, 277.

Bruce, D. (1920). f. Hyg. (Camb.), 19, 1.

Cox, C. A., Knowelden, J., and Sharrard, W. J. W. (1963). Brit. med. f., 2, 1360 .

Dienes, L. (1950). Proc. Soc. exp. Biol. (N.Y.), 75, 412.

Evans, D. G., Fuller, A. T., and Walker, J. (1945). Lancet, 2, 336.

Filler, R. M., and Ellerbeck, W. (1960). f. Amer. med. Ass., 174, 1.

Garrod, L. P. (1958). f. roy. Army med. Cps, 104, 209.

Lennert-Petersen, O. (1954). Acta path. microbiol. scand., 35, 591.

Lindberg, R. B., and Newton, A. (1954). In Antibiotics Annual, 1954-55, p. 1059, edited by $H$. Welch and F. Martin-Ibañez. Medical Encyclopaedia Inc., New York, U.S.A.

McDonald, R. T., Chaikof, L., and Truant, J. P. (1960). Surg. Gynec. Obstet., 110, 702.

Novak, M., Goldin, M., and Taylor, W. I. (1949). Proc. Soc. exp. Biol. (N.Y.), 70, 573.

Scheibel, I., and Assandri, J. (1959a). In Proceedings of an International Symposium of Immunology, p. 381. Opatija, Yugoslavia.

- (1959b). Acta path. microbiol. scand., 46, 333.

Smith, J. W. G. (1964a). Brit. med. f., 1, 373.

(1964b). In Proceedings of the Ninth International Congress of Microbiological Standardization, Lisbon, 1964. Karger, Basle/New York. In press.

Welch, H. (1948). In Methods in Medical Research, 1, pp. 1-65, edited by V. R. Potter. Year Book Publishers, Chicago.

\title{
Prodromal Phase of Measles: Some Neurophysiological Studies
}

\author{
G. PAMPIGLIONE,* M.D., M.R.C.P.
}

\section{Brit. med. F., 1964, 2, 1296-1300}

Measles is one of the most widespread diseases in the world, and in the United Kingdom it reappears in large epidemics every two years, though more of ten in small epidemics. In other countries yearly epidemics are reported (Morley, Woodland, and Martin, 1963). Probably the most striking feature in the course of measles is the appearance of the rash. The illness, however, as recognized by the patient, his relatives, and the medical practitioner, begins a few days before the appearance of the rash. As recognized by the virologist it begins even earlier (Enders, 1962). Very little is known about the cause of the rash and why it becomes established at a particular phase of the illness, during which the virus disappears rapidly.

The mechanisms responsible for some of the "complications" of measles are poorly understood, although it is generally recognized that each epidemic may differ from others. The figure of about $0.9 / 1,000$ for the neurological complications of measles, calculated by Miller, Stanton, and Gibbon (1956) as the average for 15 epidemics, is not grossly different from the figure given by Robbins (1962) and by La Boccetta and Tornay (1964). However, in a recent survey in the United Kingdom, Miller (1964) suggests a figure of 4/1,000 for all the neurological complications of measles during the 1963 epidemic. The overall mortality in cases of measles encephalitis collected from the literature reported by Miller et al. (1956) varied from $27 \%$ to $15 \%$. A mortality of $26 \%$ has been reported by Holler (1964) out of his 30 cases of measles encephalitis in children.

In the course of an epidemic of measles in England in 1963 neurophysiological studies were made on a total of 104

\footnotetext{
* Physician, Department of E.E.G. and Clinical Neurophysiology, the Hospital for Sick Children and the Royal Free Hospital, London.
}

patients. While the bulk of this work was carried out during and after the appearance of the rash and will be reported elsewhere (Pampiglione, Ramsay, Williams, and Young, 1964), the present paper describes some electroencephalographic (E.E.G.) observations made on a group of children who were examined a few days before rather than after the appearance of the measles rash. This study was made to assess the possible extent of the alteration of cerebral function in apparently uncomplicated cases of measles, during a period of the disease-the " prodromal phase"-which has until now been incompletely studied.

\section{Material}

In view of the fact that the incubation period of measles does not usually exceed 14 days, children who had E.E.G. studies two weeks or more before the appearance of the rash were not included in the present investigation. A total of 22 children had E.E.G. studies during the incubation period-that is to say, between 1 and 14 days before the appearance of the rash-and could be re-examined either during or after the end of the disease. The material was collected in the course of various E.E.G. studies in children as follows (see also Chart):

(a) Five normal children, known measles contacts (1963 epidemic), admitted to hospital (Coppetts Wood) during the prodromal phase for social reasons. These had E.E.G.s either two days or one day before the rash appeared and were retested during and after the rash.

(b) Eight normal children studied during a survey in London day nurseries (Medical Research Council project). These were tested from 11 to 3 days before the rash appeared (1963 epidemic) and retested once or twice one to three months after the rash. 
(c) Two children, known measles contacts (1963 epidemic), referred because of "febrile convulsions" (Great Ormond Street) who developed the rash three and four days respectively after their first E.E.G. and were retested during and after the rash.

(d) Seven children who had been admitted to hospital (Great Ormond Street) for some long-standing neurological condition. These children had one or more E.E.G.s from 14 to 1 day before the appearance of the rash (either in the 1961 or 1963 epidemic) and were retested during and after the rash.

To the 22 cases listed above, four other children were added who developed cough, fever, and conjunctivitis, and two had an acute neurological illness, with either prolonged drowsiness and stiff neck or an isolated seizure. They were measles contacts but did not develop a rash. Their illnesses occurred during the 1963 epidemic of measles.

The E.E.G.s were taken with a technique previously described (Pampiglione, Young, and Ramsay, 1963) with portable 8-channel apparatus (Offner type $T$ ). No sedation was given to any of the children, and all records were taken during the waking state, and also, whenever possible, during spontaneous drowsiness and sleep.

\section{Results}

The criteria for the recognition of gross E.E.G. abnormalities in children with a cerebral illness are generally agreed upon, but the criteria of normality and those of borderline abnormality, both in the United Kingdom and abroad, are often left to the experience of the individual workers, as is the case for many physical signs in medicine. In the present paper, rather than embarking on a lengthy statement of criteria of normality, a different procedure has been followed. A comparison was made between the E.E.G.s taken in each child, on the one hand, during the incubation and prodromal phases of measles, and, on the other hand, the E.E.G.s taken either before or after the end of the attack of measles. The occurrence of either similarity or change was noted between the E.E.G. taken in the incubation period and the other E.E.G.s. Particular features and the distribution of both abnormal and "unusual" E.E.G. activities seen in the prodromal phase were studied.

It was noticed that in most patients tested more than five days before the appearance of the rash, no substantial E.E.G. change occurred, in comparison with the records taken some weeks after the measles, provided no gross neurological complications had occurred in the meantime. In the patients tested one to four days before the occurrence of the rash, a moderate to severe excess of slow activity occurred in the E.E.G. whether or not some neurological trouble had occurred in the prodromal phase (Fig. 1, Fig. 2, Fig. 3). In those patients in whom it was possible to repeat E.E.G.s at fairly short intervals just before, during, and after the rash it was noticed that the severity of the E.E.G. abnormality seen in the prodromal phase might diminish, on occasion, even before the disappearance of the rash, as illustrated in Fig. 4.

\section{Discussion and Conclusions}
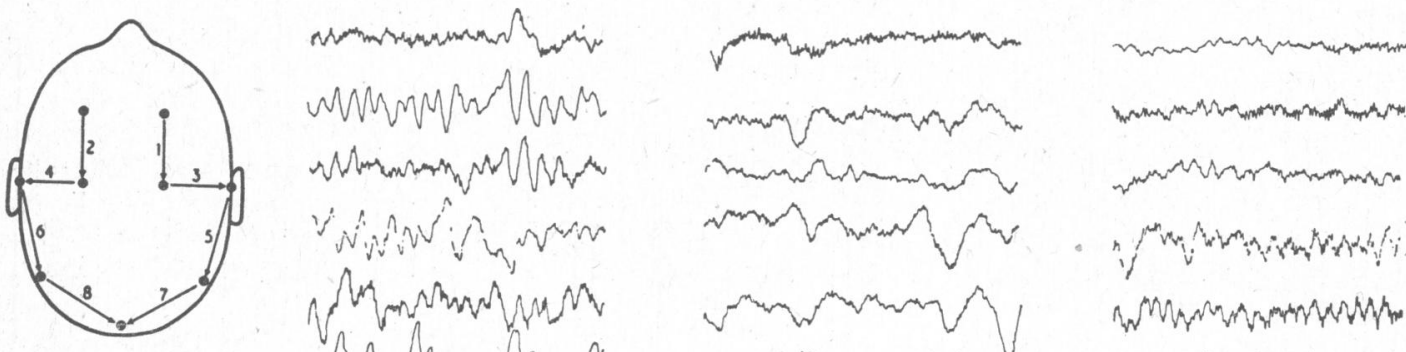

(n)

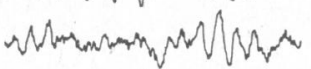

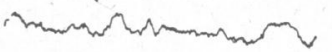

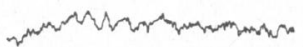

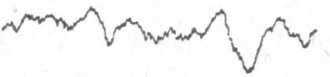
is

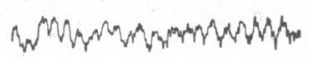

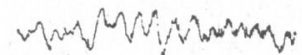

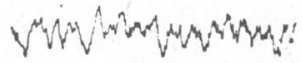
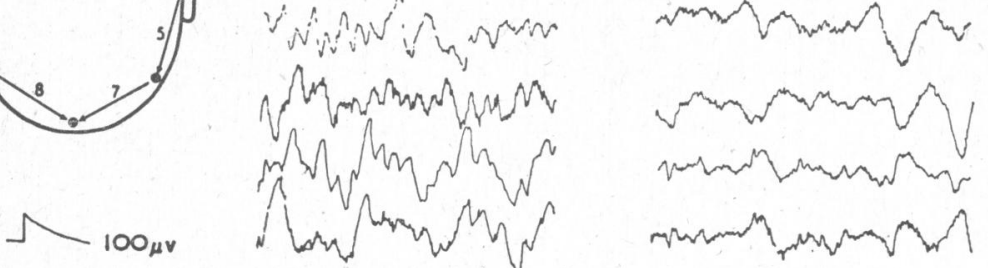

beginning of measles)

FIG. 1.-This boy, aged 2, had his first E.E.G. (A) on 12 July 1963 (the day before the beginning of measles) when his temperature was $100.5^{\circ} \mathrm{F}$. ( $\left(38.1^{\circ}\right.$ C.). The increase in irregular slow activity persisted on 15 July
during the rash (B), more obvious over the posterior than over the anterior half of the head, but had disappeared during the rash (B), more obvious over the posterior than over the anterior half of the head, but had disappeared
completely by the time the rash had faded (C) on 19 July. His temperature reached $102^{\circ} \mathrm{F}$. (38.9 $\mathrm{C}$.) on completely by the time the rash had faded $(\mathrm{C})$ on 19 July. His temperature reached but remained below $99^{\circ} \mathrm{F}$. $\left(37.2^{\circ}\right.$ C. $)$ from the afternoon of 14 July onwards. 
no gross alterations of plasma electrolyte levels were found. In contrast with a common belief, the absence, presence, type, or severity of fever did not appear to be an important factor in relation to the onset and severity of the E.E.G. abnormalities. Moreover, the E.E.G. alterations seemed to precede the appearance of fever in some cases. The rise in temperature not only was mild in all our patients reaching $101^{\circ} \mathrm{F} .\left(38.3^{\circ}\right.$ C.) (exceptionally $102^{\circ} \mathrm{F}$; $38.9^{\circ}$ C.) but usually lasted only two to three days. The great majority of the E.E.G.s showing considerable excess of slow activity in the prodromal phase were taken when the child's temperature was well below $101^{\circ}$ F. $\left(38.3^{\circ}\right.$ C. $)$.

From the literature it is readily appreciated that severe clinical changes, such as prolonged seizures, coma, or paralysis, in the course of measles, should be considered definite evidence for the diagnosis of a " neurological complication" whether occurring before, during, or after the rash. However, in practice, and particularly in the age-group most affected by measles in the United Kingdom, it seems probable that mild forms of C.N.S. involvement might be more common than appears from the published statistical studies. In children, for example, fever, conjunctivitis, cough, photophobia, restlessness, malaise, tenderness of head, neck, limbs, and a marked change of mood are part of the usual symptoms occurring some two to four days prior to the appearance of the measles rash. Some degree of drowsiness, occasional pro-

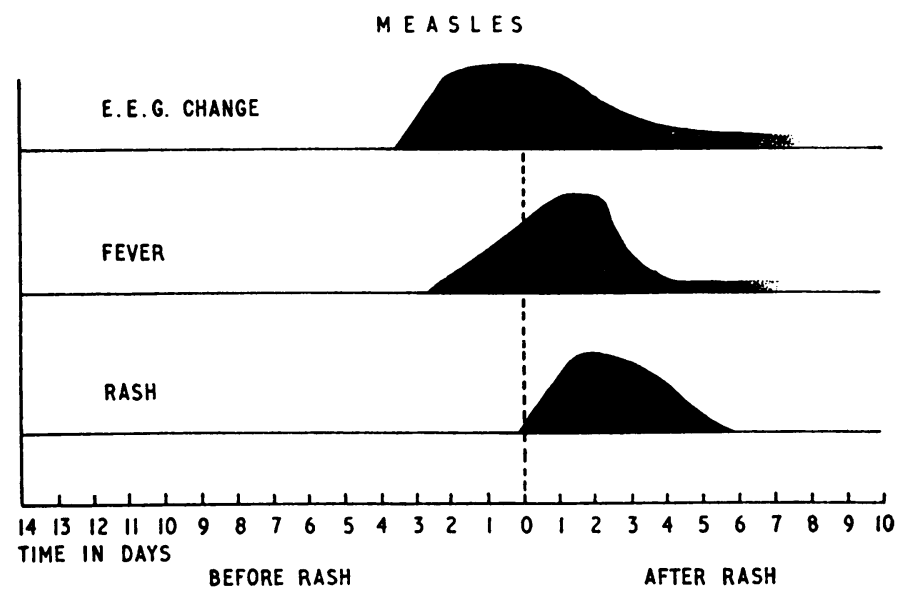

FIG. 4.-Schematic impression of the time relationships in the appearance of E.E.G. changes, fever, and rash in measles.

longed crying, and slight neck stiffness during this period are not regarded as rare symptoms, although possibly an indication of mild neurological complication. Prolonged sleepiness, short periods of confusion, or an isolated convulsion are not considered by many paediatricians as either exceptional phenomena preceding the measles rash or evidence of encephalitis in young children, although a different view may be held if these phenomena occur after the end of the rash. "Shivering fits or, in young children, convulsions may occur during the prodromal stage ; these, however, are generally slight and of short duration. In some epidemics the patient during the prodromal stage remains in a dull somnolent state" (Williams, 1906). It seems, therefore, that certain symptoms might not be included in some of the surveys on " neurological complications of measles," when the main emphasis is based on the occurrence of prolonged seizures, or gross confusion, or coma and severe motor trouble.

Gibbs, Gibbs, Carpenter, and Spies (1959), in a very interesting study of "uncomplicated" childhood diseases, found that $51 \%$ of their 680 patients with measles showed "an abnormally slow E.E.G."

PIG. 3. - This girl, aged 4, had her first E.E.G. (A) on 20 February, 1963, when her temperature fluctuated between 99 and $100^{\circ}$ F. (37.2 and $37.8^{\circ}$ C.). She developed measles rash on 22 February, when her temperatur reached for a few hours $101^{\circ}$ F. ( $38.3^{\circ}$ C.). The second E.E.G. (B) was taken on 23 February, when this child' temperature had returned to below $98^{\circ} \mathrm{F}$. (36.7 $7^{\circ} \mathrm{C}$.). The excess of slow activity had diminished by the end of the rash (C) on 27 February, and very little residual slow activity was seen six months later (D) on 21 August. 
during or after the rash. No information is given in their paper about any study that might have been extended to the prodromal phase of measles. Both in that paper and in a more recent one (Gibbs, Gibbs, Spies, and Carpenter, 1964) it is suggested that particular types of E.E.G. abnormalities might indicate some likelihood of particular clinical sequelae.

During the epidemic of 1963 it was rather puzzling to find in our material that peculiar E.E.G. alterations should be a common feature in the prodromal phase of measles at a time when a number of poorly understood clinical phenomena occurred, such as malaise, restlessness, irritability, marked mood change, headache, and the occasional seizure. The E.E.G. changes (with individual variations, partly related to age) occurred in all children of this series examined in the prodromal phase and showed some similarities. There was a poverty or total lack of the rhythmic activities normally expected in the appropriate age-group. A considerable amount of irregular activity at about $1 \frac{1}{2}$ to 3 per second was common. This irregular slow activity was only slightly affected by noises and eyeopening ; but it tended to diminish during sleep (see Gibbs et al., 1959), only to reappear on waking up. The slow activity, though generalized, was more obvious over the posterior than over the anterior half of the head, even in the apparently uncomplicated cases, often with a somewhat variable lateralization. No spikes and no complex wave forms or other more organized discharges were seen either during the prodromal phase or during the rash, even in the two children of our series who had a seizure during this phase (see Fig. 2). In these two children there were only minor differences in degree and distribution of the excess of irregular slow activity in comparison with the E.E.G.s of children seen in the same period, who had apparently uncomplicated measles (see Figs. 1 and 3). In view of these findings it is conceivable that the percentage of abnormal E.E.G.s reported by Gibbs et al. (1959) during " uncomplicated measles might increase if the records were all taken either on the first day of the rash or during the prodromal phase.
F1G. 6.-This child, aged 22 months, had had a number of E.E.G.s because of a syndrome of infantile spasms and mental and motor retardation. An E.E.G. taken i February 1963, nine days before the beginning of the Jus (A) showed features already seen in previous rash (A), showed features already seen in previous E.E.G.s in this child, including irregular slow components and a number of short-duration spikes and sharp waves. The second E.E.G. (B) taken on 14 February, when this child's temperature was $101^{\circ} \mathrm{F}$. ( $\left(38.3^{\circ} \mathrm{C}\right.$.), showed an increase in 2-3 c/s waves and a disappearance of the discharges. The measles rash began the following day, 15 February. In the E.E.G. taken several weeks after the measles (C) the discharges had reappeared. Other changes in rhythmic components might have been related to the administration of drugs.

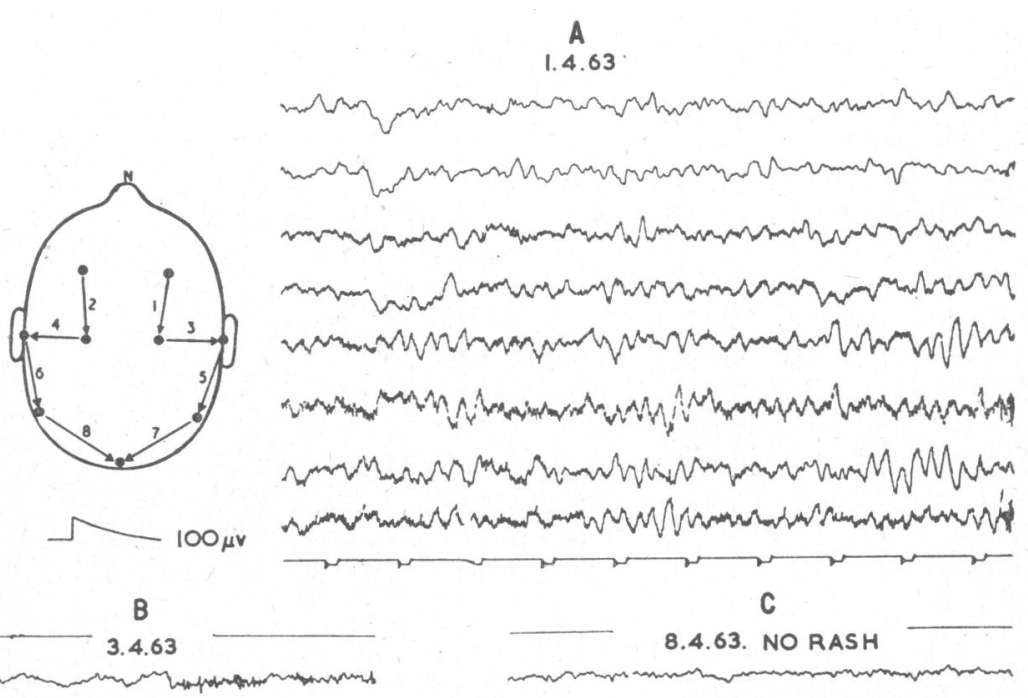

monoms

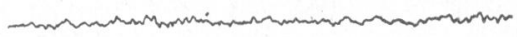

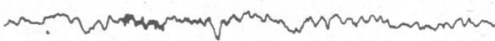

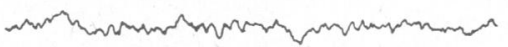

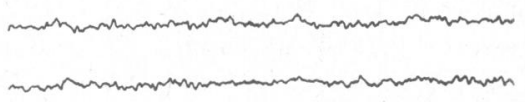

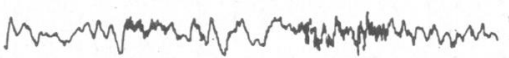

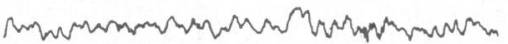

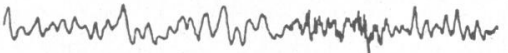

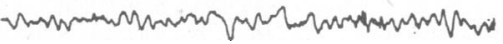

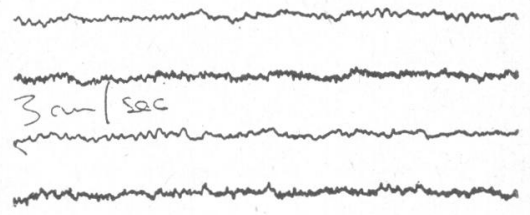

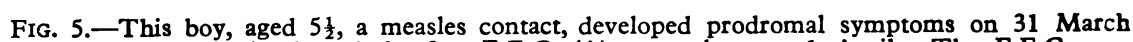
1963. He was admitted, and the first E.E.G. (A) was taken on 1 April. The E.E.G. was repeated on 3 April (B) and the excess of slow components persisted. This E.E.G. change had entirely disappeared by the time of the third E.E.G. (C) on 8 April while the child was perfectly well and had developed no rash.

A $10,451 \quad 5.2 .63$

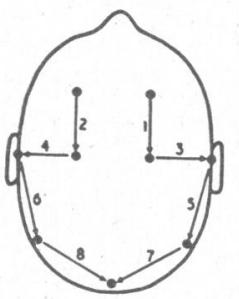

$N_{100 \mu v}$

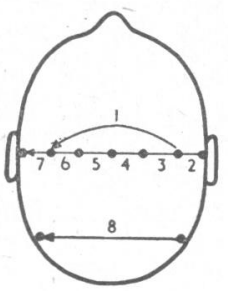

$N-100 \mu v$

C
B

$10,545 \quad 14.2 .63$

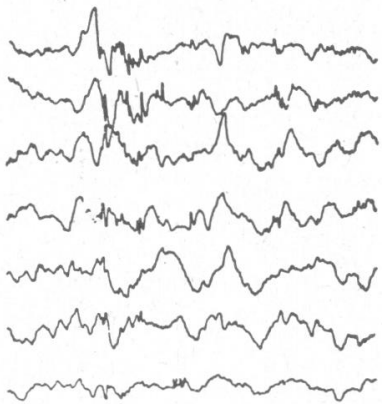

mann

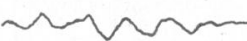
rnom manconans

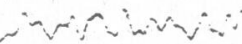
ramenting rin smencนn

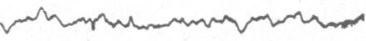
$00_{\mu v}$ $11,032 \quad 1.4 .63$

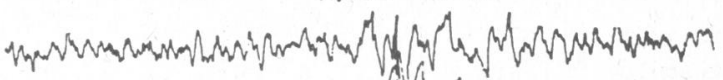

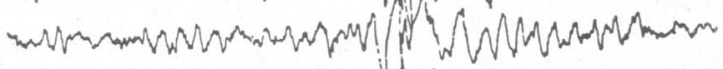

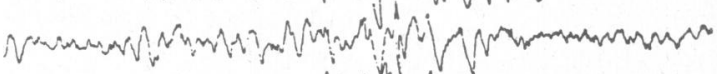

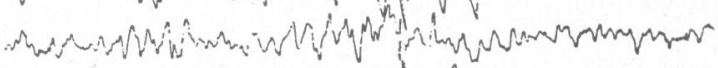

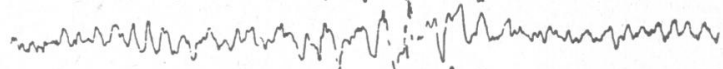

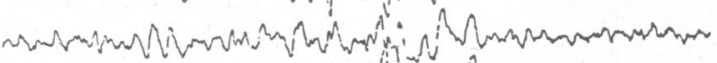

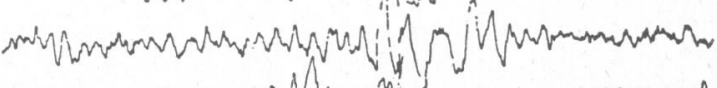

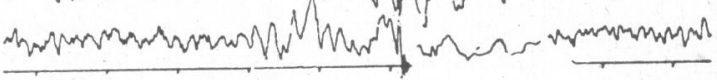


E.E.G. changes parallel to those described above were seen during the 1963 epidemic also in four other children known to be measles contacts, who developed the prodromal symptoms but did not develop a rash (Fig. 5). Two of them had been admitted to hospital for a neurological illness retrospectively considered as a mild form of acute encephalitis of unknown aetiology, and had an isolated seizure while feverish during what was thought to be the prodromal phase of "measles without a rash." In one of them both virological and immunological studies failed to support the diagnosis of measles. It is interesting to note, as an indication for further studies, that two other children with a long history of seizures and frequent discharges in their E.E.G. well before measles tended to show a diminution in paroxysmal features in their E.E.G. taken during the prodromal phase of measles, and a return to their original pattern after the rash had disappeared (Fig. 6).

Why should the electrical activity of the brain be affected so commonly in measles, and why should the E.E.G. changes precede the rash and coincide with the appearance of some of the less understood clinical phenomena of the prodromal phase ? Why, during an epidemic, should some patients, known to be contacts of measles, exhibit no rash in spite of showing other features of measles such as cough, fever, conjunctivitis, and even the peculiar E.E.G. changes? The old hypothesis that a number of pathological processes already present might be "activated" by the infection of measles is not fully proved, but may not be rejected on the meagre evidence at present available. An equally plausible hypothesis is that not only the respiratory and tegumental systems, but also the central nervous system among others might be directly affected by the virus, early in the course of measles. It would be of great interest, however, to study the changes that occur in the course of measles, in the oral and intestinal mucosa, and, particularly, in the intestinal flora. It seems possible that marked changes in intestinal absorption may occur during measles, particularly in the prodromal phase and during the enanthem even more than during the exanthem. Nutritional studies might demonstrate whether some of the effects upon the C.N.S. might be related to acute malabsorption or "toxic" phenomena, rather than direct effect of the virus in the brain. Very little seems to be known about the changes in metabolic requirements, particularly in terms of proteins, during the various phases of measles, although it is well recognized that some complications are much more common in children with poor nutrition than in well-nourished children (Taneja, Ghai, and Bhakoo, 1962 ; Morley, 1962).

The E.E.G. observations reported above may be relevant to the study and particularly to the timing of some effects of various measles vaccines, in selected subjects and particular age-groups. A correct interpretation of the causes of the E.E.G. phenomena described above must be postponed to a time when a little more is known about the physiopathological mechanisms underlying measles, this easily recognizable disease about which we still know so little.
In the meantime, however, it would be desirable to carry out more extensive studies during the prodromal phase of measles, with combined clinical, metabolic, neurophysiological (E.E.G.), and psychological documentation, according to a timely plan for the next epidemic in various parts of the world.

\section{Summary}

In the course of an epidemic of measles in England in 1963 E.E.G. studies were made on 22 children during the incubation period and during and after the appearance of the rash. In all the patients tested one to four days before the occurrence of the rash, a moderate-to-severe excess of slow activity was seen in the E.E.G. The severity of the E.E.G. change often increased on the first day of the rash, decreasing gradually during the ensuing days. Fever did not appear to be an important factor in relation to the onset and severity of the E.E.G. changes. Various possible factors underlying the E.E.G. changes during the prodromal phase of measles and during the rash are discussed. If these preliminary results are confirmed by the study of larger groups of children, the E.E.G. change might be regarded as part of the usual events in measles during the prodromal period as well as during the rash. The present findings might be relevant to the study of some effects of various measles vaccines in selected subjects and particular age groups.

I am indebted to Dr. A. Dudgeon, Professor Sir Alan Moncrieff, and Professor C. H. Stuart-Harris for their kind suggestions. This investigation was supported, in part, by a grant from the Medical Research Council (day nurseries survey) and, in part, by the Polio Research Fund (mobile neurophysiological unit). My thanks are due to my indefatigable E.E.G. technicians, to the Department of Medical Illustration (Institute of Child Health), and to my colleagues, particularly to Dr. M. Ramsay, who referred their patients.

\section{REFERENCES}

Enders, J. F. (1962). Amer. F. Dis. Child., 103, 282.

Gibbs, F. A., Gibbs, E. L., Carpenter, P. R., and Spies, H. W. (1959). f. Amer. med. Ass., 171, 1050.

Spies, H. W., and Carpenter, P. R. (1964). Arch. Neurol. In press.

Holler, O. (1964). Arch. Kinderheilk., 170, 153.

La Boccetta, A. C., and Tornay, A. S. (1964). Amer. f. Dis. Child., 107, 247.

Miller, D. L. (1964). Brit. med. f., 2, 75.

Miller, H. G., Stanton, J. B., and Gibbons, J. L. (1956). Quart. F. Med., $25,427$.

Morley, D. C. (1962). Amer. F. Dis. Child., 103, 230.

- Woodland, M., and Martin, W. J. (1963). F. Hyg. (Lond.), 61, 115.

Pampiglione, G., Ramsay, M., Williams, M., and Young, S. (1964). In preparation.

_ Young, S. E. J., and Ramsay, A. M. (1963). Brit. med. F., 2, 1300.

Robbins, F. C. (1962). Amer. F. Dis. Child., 103, 266.

Taneja, P. N., Ghai, O. M. P., and Bhakoo, O. N. (1962). Ibid., 103, 226.

Williams, D. (1906). In T. C. Allbutt and H. D. Rolleston's A System of Medicine, vol. II, part I, p. 389. Macmillan, London. 